

Original Research Article https://doi.org/10.20546/ijcmas.2017.605.101

\title{
Prevalence of Betahemolytic Streptococci other than Group A in a Tertiary Health Care Centre
}

\author{
Illakya, V. Sangamithra*, Shabana Praveen and Radha Madhavan \\ Department of Microbiology, SRM Medical College \& RI, Chennai, India \\ *Corresponding author
}

\begin{tabular}{|c|c|}
\hline & A B S T R A C T \\
\hline & ositive spherical bacteria (cocci) that characteristically form \\
\hline & $\begin{array}{l}\text { pairs and chains during growth. They are widely distributed in nature. Some are members of } \\
\text { the normal human flora for e.g. commensal Streptococci of the oral cavity, are common }\end{array}$ \\
\hline $\begin{array}{l}\text { Betahemolytic } \\
\text { Streptococci, }\end{array}$ & causes of sub-acute bacterial endocarditis, others are associated with important human \\
\hline $\begin{array}{l}\text { Bacterial } \\
\text { endocarditis. }\end{array}$ & $\begin{array}{l}\text { Streptococcus species is uniformly susceptible to penicillin. But macrolides remains drug of } \\
\text { choice for patient allergic to penicillin. Our aim was to study the prevalence of betahemolytic }\end{array}$ \\
\hline Article Info & infections. A total of 366 positive cultures were taken from various samples of patients \\
\hline $\begin{array}{l}\text { Accepted: } \\
\text { 12 April } 2017 \\
\text { Available Online: } \\
10 \text { May } 2017\end{array}$ & $\begin{array}{l}\text { attending the Medicine and Paediatric outpatient department. Indiscriminate use of } \\
\text { Macrolides for respiratory tract infection in the private practices has resulted in very high } \\
\text { percentage of macrolide resistant among Streptococci in the population studied Increasing } \\
\text { antimicrobial resistance is becoming a serious international problem in both hospital and }\end{array}$ \\
\hline & Macrolides in several countries \\
\hline
\end{tabular}

\section{Introduction}

Beta hemolytic Group C Streptococci (GCS) account for about $5 \%$ of all cases of Streptococcus infection in adult human. These often look like Group A Streptococci on Blood Agar and are beta hemolytic Streptococci of this group are predominantly animal pathogens. The bacteria cause a condition known as "pharyngitis", an inflammation of the mucous membrane of the pharynx or throat which was formerly called Strep throat. The symptoms in humans can range in severity from very mild to incredibly severe. Aside from the sore throat, it can produce low to high fever, neck swelling, enlarged tonsils, and hoarseness and even acute to moderate nausea.
More severe symptoms included arthritis, pneumonia and even bacteremia all of which could lead to toxic shock (Ashton H Minty, Information on Beta Strep Group C)

On the other hand, Group G Streptococci (GGS) was identified in 1935 by Lancefield and Hare, as a part of normal flora of the pharynx, GIT, Genital tract and skin. And also it is present as commensal in throats of monkeys or dogs (CDC, Emerging infectious disease) It is usually not inhibited by Bacitracin. In recent years, GGS have been reported to cause a variety of human infections, such as, sore throat, pharyngitis, cellulitis, meningitis, infection of the heart 
valves (endocarditis) and sepsis. Blood stream infection (bacteremia) due to GGS has been related to underlying conditions, such as alcoholism, diabetes mellitus, malignancy, intravenous substance abuse or breakdown of the skin. The spectrum of GGS infections ranged from mild skin and soft tissue infection (34 [46\%]) to invasive diseases, including urogenital infection (7 [10\%]); lower respiratory tract infection (7 [10\%]); pharyngitis (6 [8\%]); endocarditis and catheter infection (5 [7\%]); and others (14 [19\%]), such as peritonitis, pelvic abscess, rectal abscess, and septic arthritis. Four of the 6 persons with pharyngitis were assumed to be colonized with the organism. Eight $(24 \%)$ of 34 skin and soft tissue infections were associated with bacteremia, $5(15 \%)$ with osteomyelitis, and $20 \quad(59 \%)$ with polymicrobial infections (San Wong, 2009).

Reported mortality rates for patients with GGS bacteremia also vary ranging from 5\% to30\% (Liao et al., 2008). It is an important cause of endocarditis, septicaemia and septic arthritis.

Group C and Group G Streptococci have caused well documented epidemics of acute pharyngitis in children, but the importance of these organisms in causing endemic or sporadic pharyngitis is uncertain. The heterogenicity of GCS and GGS may obscure the role of certain subtypes, such as the largecolony-forming strains of group C (Strep. dysgalactiae subsp. equisimilis) or Group $\mathrm{G}$ in endemic pharynigitis (Zaoutis et al., 2004).

They cause isolated exudative or common source epidemic pharyngitis and cellulitis, indistinguishable clinically from GAS disease (Arnold N Weinberg) Beta hemolytic GCS and GGs and hemolysin deficient variants cause epidemics of exudative pharyngitis pharyngotonsillitis (Gail William). Some strains contain fibrinolysin and streptolysins and infections can stimulate antistreptolysin $\mathrm{O}$ titres (ASO), similar to GAS.

Patients who are exposed to farm animals, zoo animals or unpasteurized milk products are at an increased risk for GCS or GGS, since they are inhabitants of horses, cows, swine's, sheep, goats and guinea pigs. Rapid diagnosis of pharyngeal carriage of GCS and GGS strains, that lead to glomerulonephritis, toxic-shock syndrome and Rheumatic fever, may prevent unnecessary death and disability (Williams, Gail)

The majority of GCS and GGS strains demonstrate in vitro susceptibility to penicillin's, vancomycin, erythromycin and cephalosporins. Antimicrobial tolerance, defined as minimum bactericidal concentration (MBC) 32 or more times higher than the MIC, among GCS and GGS has been reported for penicillin and other agents. Only a few clinical isolates have been reported to exhibit tolerance of vancomycin (Theoklis Zaoutis et al., 1999).

Different mechanisms of erythromycin resistance predominate in Group $\mathrm{C}$ and $\mathrm{G}$ Streptococcus isolates collected from 1992 to 1995 in Finland. Of the 21erythromycin resistant GCS and 32 erythromycin resistant GGS isolates, $95 \%$ had the mef A or mef E drug efflux gene and 94\% had the erm TR methylase gene respectively (Janne Katraja, Helena Seppala et al., 1998).

According to the studies done in France, the Group A and G Streptococci were typically susceptible to penicillin G (MIC 0.003 to $0.5 \mu \mathrm{g} / \mathrm{ml}$ ) and relatively resistant (MIC 5 to $250 \mu \mathrm{g} / \mathrm{ml})$ to aminoglycoside antibiotics (naturally low-level resistance) (Thea Horodniceanu et al., 1982). Penicillin resistance was not yet demonstrated in A, C or G, beta-hemolytic Streptococci. All betahemolytic Streptococci included in this study 
were susceptible in vitro to penicillin, chloramphenicol and ceftriaxone in Argentina (Lopardo).

The factor most directly associated with the increase in antimicrobial resistance is the high level of antibiotic consumption among the population. Nonetheless the final cause of higher or lower prevalence of antimicrobial resistance depends on the circulatory clones (Emilio perez-Trallero et al., 2007).

The main objective includes to study the prevalence of betahemolytic Streptococci causing various infections like respiratory and skin infections. To study the antibiotic resistance pattern among the isolates. And to detect the phenotypic pattern of Macrolide resistance by D-test.

\section{Materials and Methods}

A prospective study was under taken which included all age group attending general medicine OPD and Pediatric OPD of SRM Medical College Hospital from the month of March 2011-2012.Patients with symptoms of respiratory and pyogenic infection were included in study. Patients who were already on antibiotics were not included. Various samples like throat swab, Sputum, Blood, Pus, Tracheal aspirate, Bronchial wash, Bronchio-alveolar lavage etc. were collected with detailed case history. The samples were subjected to Gram staining, Gram positive cocci in short chains and pairs were observed in smear. They were then plated onto Blood agar plates-to observe haemolysis and Chocolate agar plates to observe bleaching and MacConkey agar plates.Basic biochemical tests for identification like Catalase, Bacitracin sensitivity, Pyrase test were performed for presumptive identification of beta hemolytic Streptococci, some Beta hemolytic colonies other than GAS also gave Bacitracin positivity. Further confirmation was done by performing latex slide agglutination grouping tests using kits (e.g.: Hi strep latex test kit from Hi Media)

\section{Antibiotic susceptibility}

Antibiotic susceptibility of the isolates was done by Kirby-Bauer method of disk diffusion using Blood agar plates adjusting the turbidity of the inoculum to McFarland 0.5 standard, the bacterial isolate was grown in Brain heart infusion broth and Todd-Hewitt broth. The antibiotic discs namely Bacitracin (0.04 units), Penicillin(10 units), Cephalothin (30 microgram) Clindamycin (2 microgram), Erythromycin (15 microgram), Cotrimoxazole (30 microgram), amoxyclav (20 and 10 microgram), ofloxacin, ceftriaxone (30 microgram), cefuroxime (30 microgram) were included to study the antibiotic pattern. Commercially available antibiotic discs were checked for quality using standard strains and then used for the test. Antimicrobial impregnated disks were placed on the Blood agar plates with the help of sterile forceps. Disks must be placed evenly with $24 \mathrm{~mm}$ distance from center to center of each disk. Invert the plates and incubate them at 18-24 hours at $37^{0} \mathrm{C}$ with $10 \%$ carbon dioxide.

The plates are examined after overnight incubation. Zone of diameter is measured using calipers or a ruler. They were then compared with the NCCLS (CLSI) published guidelines.

\section{Double disk diffusion test (D-test)}

Testing of Streptococcal isolates with erythromycin and clindamycin disks applied closed together can often yield phenotypic information, although it is not always possible to differentiate between phenotypes using this method. D-test is performed for the detection of inducible clindamycin resistance. The $\mathrm{erm}$ genes encode resistance to the macrolides and lincosamides. 
The gold standard for diagnosis of inducible resistance is the genotyping.

(CLSI Guide line, Wayne, pa: clinical and lab standard institute 2010)

The Clinical and Laboratory Standard Institute (CLSI) recommends disk diffusion or broth micro dilution testing for susceptibility testing of Streptococci.To ensure accurate results, laboratories should include a test for detection of inducible clindamycin resistance. The double disk diffusion method (D-zone test) is recommended for testing erythromycin resistant and clindamycin susceptible strain. A sterile cotton swab is dipped into a suspension of 18-24 hours growth of the organism in Todd-Hewitt or Brain Heart Infusion broth equal to a $0.5 \mathrm{McFarland}$ turbidity standard within 15minutes of adjusting the turbidity at room temperature. The swab should be rotated several times and pressed firmly on the inside wall of the tube above the fluid level. Use the swab to inoculate the entire surface of a plate of Mueller-Hinton agar with 5\% sheep blood.

After the plate is dry, use sterile forceps to place a clindamycin $(2 \mu \mathrm{g})$ disk and an erythromycin $(15 \mu \mathrm{g})$ disk $12 \mathrm{~mm}$ apart for Dzone testing (Note - this differs from recommended $15-26 \mathrm{~mm}$ for Staphylococci and a disk dispenser cannot be used to place disks on the plate for Streptococci testing (Paul H. Edelstein, 29 Apr, 2003) Incubate inoculated agar plate at $35^{\circ} \mathrm{C}$ in $5 \% \mathrm{CO} 2$ for 20-24 hours. Isolates with blunting of the inhibition zone around the clindamycin disk adjacent to the erythromycin disk (D-zone positive) should be considered to have inducible clindamycin resistance and are presumed to be resistant.

There are three types zone were seen in this. They are, iMLSB-the clindamycin zone is blunted towards the erythromycin because the erythromycin induces clindamycin resistance. cMLSB- No zone around either erythromycin or clindamycin because erm gene is fully expressed all times. $\mathrm{M}$ - type- no change in the clindamycin zone induced by erythromycin because mef does not pump out clindamycin regardless of erythromycin presence.

\section{Results and Discussion}

In our study, 366 positive cultures were taken from various samples; $49.63 \%$ were sputum, $36.3 \%$ were throat swab, $8.89 \%$ were pus, $2.59 \%$ were blood, $2.22 \%$ were tracheal aspirate, $0.37 \%$ was bronchial wash. A Total number of 297 Beta hemolytic Streptococci were isolated of which the rate of prevalence of GAS infection was the highest $(41.41 \%)$ among the sample collected, followed by GGS (14.82\%) and GCS (11.45\%) (Table 1).

There is rise in GCS and GGS infection compared to prior studies and these results highlights the need to consider these as potential pathogen whenever isolated from cultures.

The rate of prevalence of Pneumococcal infections is also high (18.48\%) compared to other study; of which $63.77 \%$ from sputum, $33.33 \%$ from throat swab, $1.45 \%$ from blood, $1.45 \%$ from tracheal aspirate.

Infection caused by GAS, GCS and GGS was seen more common in the age group of 45-60 years, whereas Pneumococcal infections were more in the age group of 15-30years.

The resistance pattern for other antibiotics in GAS were as follows, erythromycin 64\%, penicillin $32.25 \%$, clindamycin $27.05 \%$, cotrimoxazole $94.26 \%$, cephalothin $11 \%$, amoxyclav $2.46 \%$, ofloxacin $22.95 \%$, ceftriaxone $1.67 \%$, cefuroxime $2.46 \%$.In GCS resistance were, erythromycin $41 \%$, penicillin 
$44.12 \%$, clindamycin $14.71 \%$, cotrimoxazole $85.29 \%$, cephalothin $23.53 \%$, amoxycalv $0 \%$, ofloxacin $32.35 \%$, ceftriaxone $11.77 \%$, cefuroxime $2.94 \%$.In GGS antibiotic resistance pattern were, erythromycin $41 \%$, penicillin $34.09 \%$, clindamycin $18.18 \%$, cotrimoxazole $97.73 \%$, cephalothin $13.64 \%$, amoxyclav $9.09 \%$, ofloxacin $18.18 \%$, ceftriaxone $4.55 \%$, cefuroxime $2.27 \%$. In Pneumococci, resistance was as follows: erythromycin $35.29 \%$, penicillin $51.47 \%$, clindamycin $7.35 \%$, cotrimoxazole $92.65 \%$, cephalothin $4.41 \%$, amoxycalv $5.88 \%$, ofloxacin $34 \%$, ceftriaxone $5.88 \%$, cefuroxime $8.82 \%$.

In our studies the percentage of macrolide resistance among Beta Hemolytic Streptococci was $41.29 \%$ by MIC method. In GAS $64 \%$, in GCS $41 \%$, in GGS $41 \%$ by MIC method, this is alarming high as compared to the many of the earlier studies especially in India. It may be because of the difference in population included in the study, as this a hospital based study, where only positive samples were taken, rather than a community based study done by others.

According to our studies, in GCS, cMLS was $17.6 \%$, $M$ type was $82.35 \%$ In GGS, cMLS was $31.58 \%$, iMLS was $10.53 \%$ and M type was $57.89 \%$ resistance pattern in GAS by Dtest, cMLS was $27.85 \%$, iMLS was $13.92 \%$, $\mathrm{M}$ type was $55.69 \%$.

The results of this study were similar to the finding by Thangam Menon, which showed higher rates of $M$ type among macrolide resistant isolates. But this was opposite to the finding of Melo crustino who showed high percentage of cMLS. Our study also show a high level of iMLS, among GAS and GGS which was not reported in many of the earlier studies.

A study was conducted from March 20112012 at SRM Hospital Kattankulathur to study the prevalence of beta haemolytic Streptococci other than Group A causing various infections, like respiratory tract infections, Bacteremia, skin infections, etc., Antibiotic resistance pattern among the Streptococcus strains were also studied along with the phenotypic pattern of the macrolide resistance using D-test.

All positive cases of beta haemolytic Streptococci Streptococci other than Group A were included in this study. A detailed case history was collected for all the positive cases. Antibiotic resistance pattern of each isolate were detected.

Total samples collected was 366, 270 were positive, out of which $49.63 \%$ were sputum, $36.3 \%$ were throat swab, $8.89 \%$ were pus, $2.59 \%$ were blood, $2.22 \%$ were tracheal aspirate, $0.37 \%$ were bronchial wash. Out of 366 strains collected, Beta Hemolytic Streptococci (BHS) were 297(81.15\%), which when serotyped with antisera, 123(41.41\%) were positive with Group A antisera, 34 $(11.45 \%)$ were positive with Group C antisera, and $44(14.82 \%)$ with Group G antisera, others were non typeable.

Non typeable strains were $(32.32 \%)$ not included in our studies. All alpha hemolytic Streptococci sensitive to Optochin were included as Pneumococci, which was $69(18.85 \%)$.

All the strains were stocked for further studies. Antibiotic Sensitivity tests (AST) were done for all isolates on Mueller Hinton agar with 5\% sheep blood. All erythromycin intermediately sensitive and resistant strains were grouped as resistant strains and stocked for further study. A D test was done by using erythromycin $(15 \mu \mathrm{g})$ and clindamycin $(2 \mu \mathrm{g})$ disc kept at distance of $15 \mathrm{~mm}$ apart to detect the phenotypes of macrolide resistance among the isolates of all beta hemolytic Streptococcus species. 


\section{Prevalence of macrolide resistance}

The resistance pattern for GCS were as follows erythromycin $41 \%$, penicillin $44.12 \%$, clindamycin $14.71 \%$, cotrimoxazole $85.29 \%$, cephalothin 23.53\%, amoxycalv $0 \%$, ofloxacin $32.35 \%$, ceftriaxone $11.77 \%$, cefuroxime $2.94 \%$.In GGS the resistance pattern were as follows erythromycin $41 \%$, penicillin $34.09 \%$, clindamycin $18.18 \%$, cotrimoxazole $97.73 \%$, cephalothin $13.64 \%$, amoxyclav $9.09 \%$, ofloxacin $18.18 \%$, ceftriaxone $4.55 \%$, cefuroxime $2.27 \%$. Resistance pattern for GAS were as follows, erythromycin $64 \%$, penicillin $32.25 \%$, clindamycin $27.05 \%$, cotrimoxazole $94.26 \%$, cephalothin $11 \%$, amoxyclav $2.46 \%$, ofloxacin $22.95 \%$, ceftriaxone $1.67 \%$, cefuroxime $2.46 \%$. In studies done in New York, 14 to $34 \%$ of S. pyogenes isolates were erythromycin-resistant, and $0 \%$ to $28 \%$ was clindamycin-resistant. None of the $s$. pyogenes isolates were resistant to Penicillin

Prevalence rate of macrolide resistance of Streptococcus pyogenes from various countries have been reported as $10 \%$ in
Sweden, 17\% in Finland and 22\% in UK. Higher rates of resistance $(>50 \%)$ has been reported in Taiwan, Japan and lower $(2 \%)$ in Canada in Portugal $35.8 \%$, Turkey $3.8 \%$ and Italy $38.3 \%$. In South India, studies done by Menon et al., showed $16.2 \%$ in 2007. It may be because of the difference in population included in the study, as this is a hospital based study, only positive samples were included, rather than a community based study done by others. In D test done to detect the Phenotypic pattern of the erythromycin resistance to detect cMLS, iMLS and M type, studies done by Melo Crustino 1999, in Portugal shows cMLS as $79.6 \%$, M type as $16.7 \%$. In studies done by Thangam Menon et $a l$., in 2006 the percentage of erythromycin resistance strain showed cMLS as $26.3 \%, \mathrm{M}$ type as $73.6 \%$. Our study we included both intermediate and resistance strain for typing, According to our studies resistance pattern in GAS, cMLS was $27.85 \%$, iMLS was $13.92 \%$, $M$ type was $55.69 \%$. In GCS, cMLS was $17.6 \%$, M type was $82.35 \%$. In GGS, cMLS was $31.58 \%$, iMLS was $10.53 \%$ and M type was $57.89 \%$.

Table.1 Distribution of positives in different samples

\begin{tabular}{|l|l|l|l|l|}
\hline Specimen & GAS(123) & GCS(34) & GGS(44) & Pneumococci(69) \\
\hline Throat swab & $46(37.4 \%)$ & $12(35.3 \%)$ & $17(38.61 \%)$ & $23(33.33 \%)$ \\
\hline Sputum & $54(43.9 \%)$ & $16(47.06 \%)$ & $20(45.45 \%)$ & $44(63.77 \%)$ \\
\hline Pus & $15(12.2 \%)$ & $4(11.76 \%)$ & $5(11.36 \%)$ & 0 \\
\hline Blood & $4(3.25 \%)$ & $2(5.88 \%)$ & 0 & $1(1.45 \%)$ \\
\hline Tracheal aspirate & $4(3.25 \%)$ & 0 & $1(2.27 \%)$ & $1(1.45 \%)$ \\
\hline Bronchial wash & 0 & 0 & $1(2.27 \%)$ & 0 \\
\hline
\end{tabular}


Fig.1 A diagrammatic representation of the positive samples

$18.85 \%$

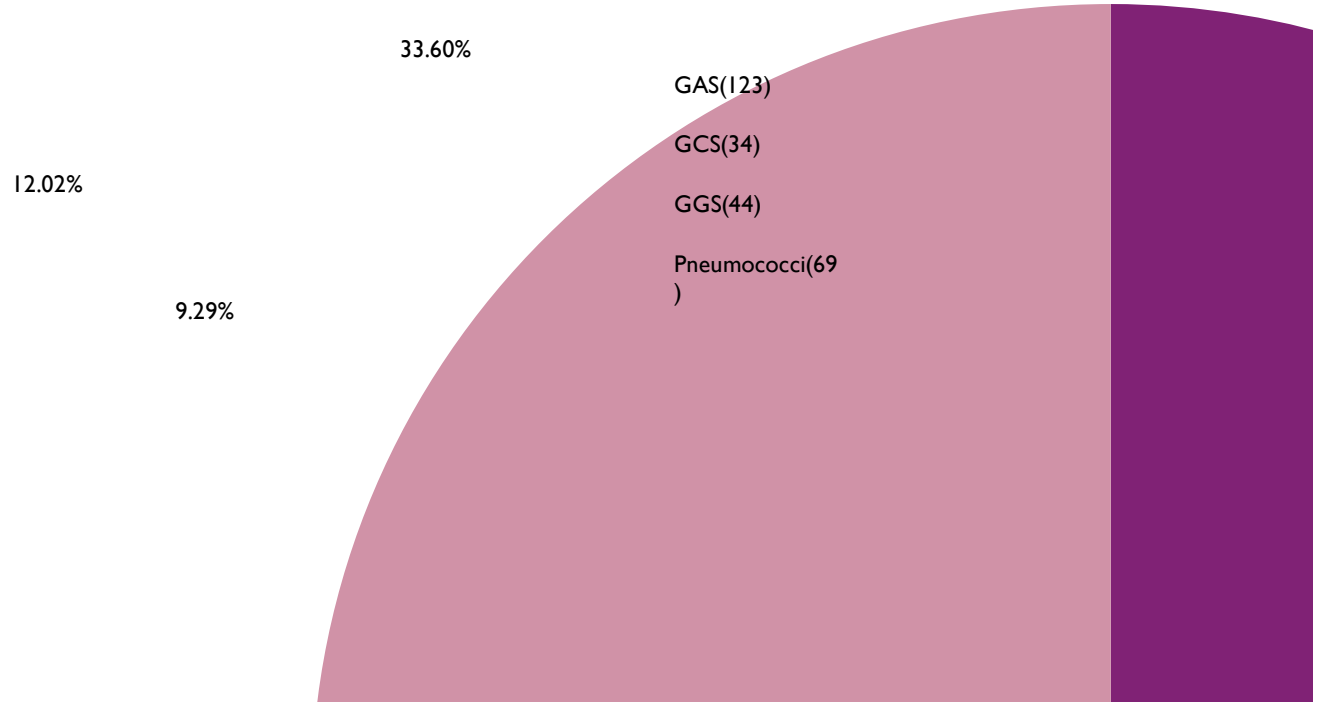

Fig.2 Graphical representation of Comparison of Age Sex ratio among total positive

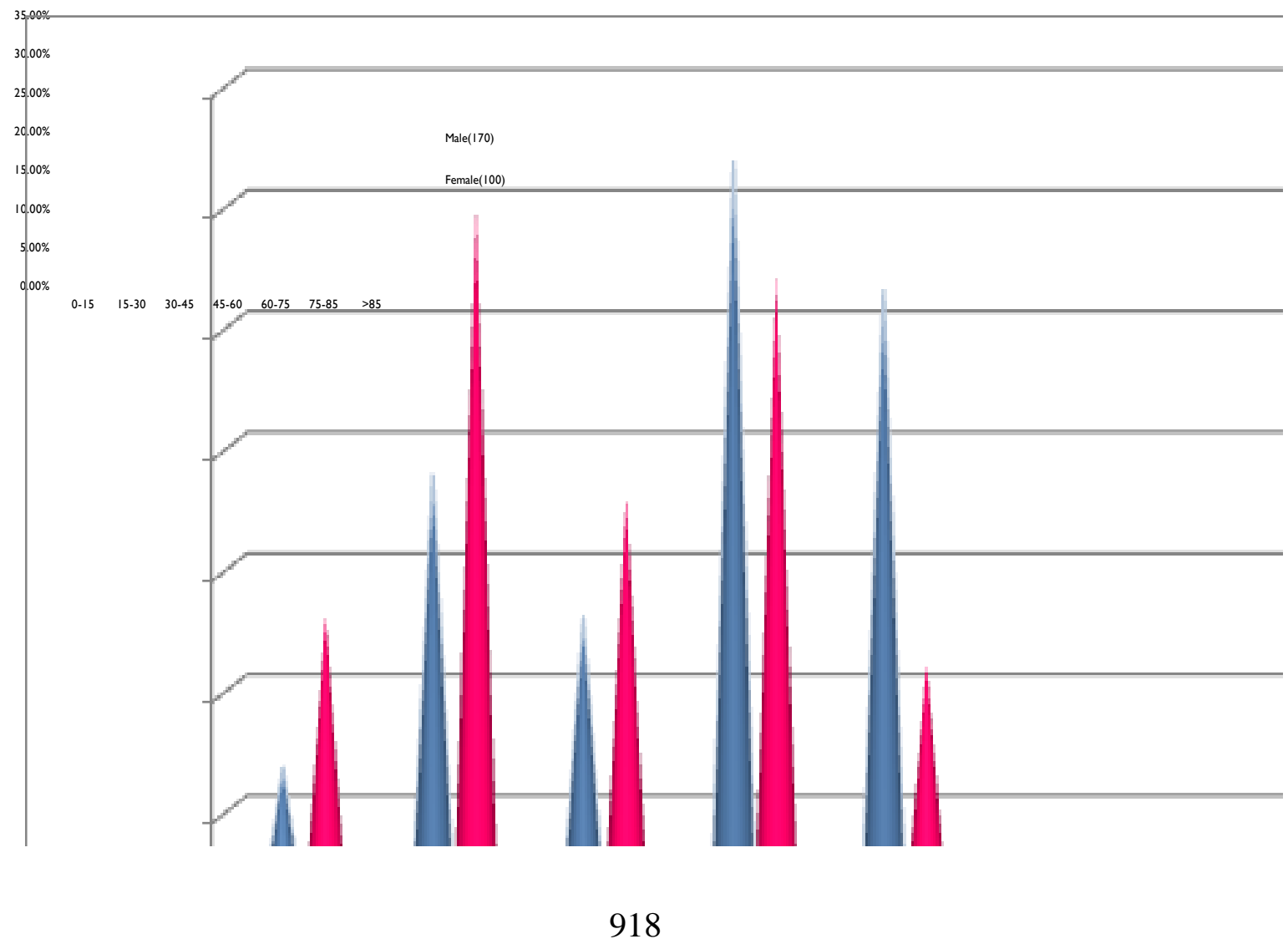


Fig.3 Graphical representation of Age wise distribution in Streptococcus species

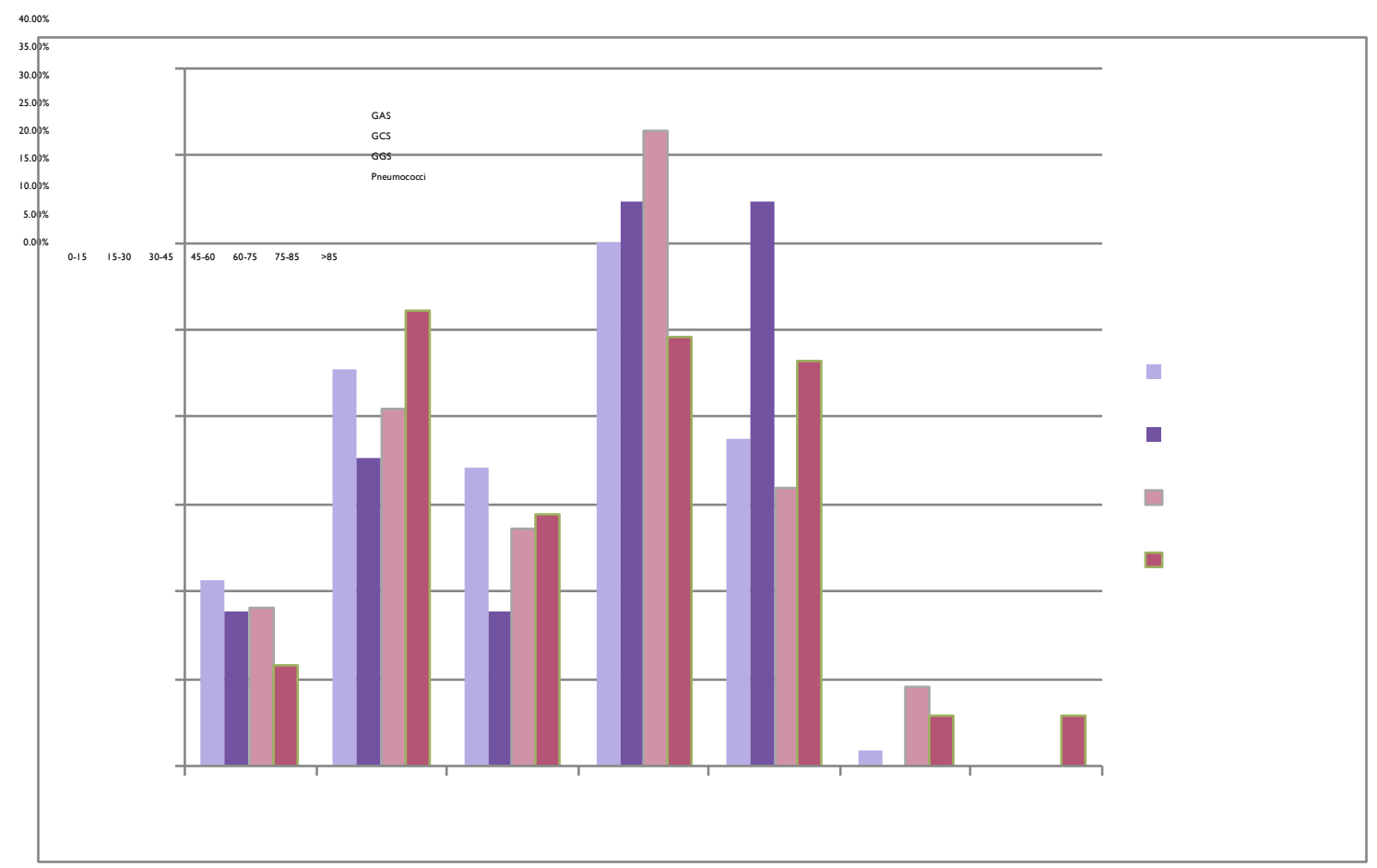

Fig.4 Resistance pattern in Streptococcus species




Fig.5 Resistance pattern in Streptococcus species (D test)



Our results were similar to the finding done by Thangam Menon, which showed higher rates of $\mathrm{M}$ type among Macrolide resistant isolates. But this was opposite to the finding of Melo crustino who showed high percentage of cMLS. Our study also show a high level of iMLS, among GAS and GGS which was not reported in many of the earlier studies.

In conclusion, thus according to our studies there is an alarming rise in GAS infection, which is of great concern. Macrolide resistances among the isolates are also higher compared to many studies. This can be explained by the over usage of macrolide for respiratory infections without proper antibiotic policies. The finding expand our knowledge of various Streptococcal infection in our population, GGS and GCS infections are on the rise which is justified, as the population studied was mostly communities living in villages closely associated with cattle and other pet animals. GAS infection are alarmingly high and Pneumococcal infections were also higher, compared to the other studies; therefore we need to insist on the importance of administration of pneumococcal vaccines wherever necessary.

Indiscriminate use of Macrolides for respiratory tract infection in the private practices has resulted in very high percentage of macrolide resistant among Streptococci in the population studied; compared to other studies. Therefore by this study we would like to highlight the necessity to do antibiotic sensitivity testing for all isolates, and limit the usage of antibiotics, whenever necessary and select the appropriate antibiotics for resistant strains.

\section{References}

Alberti, S., Garcia-Rey, M.A., Dominguez, L. Aguilar, E. Cercenado, M. Gobernado, and A. Garcia-Perea. 2003. Survey of emm gene sequences from pharyngeal Streptococcus pyogenes isolates collected in their Spain and their relationship with 
erythromycin susceptibility. J. Clin. Microbiol., 41: 2385-2390.

Alexander Tomasz. 2012. Antibiotic Resistance in Streptococcus pneumoniae, The Rockefeller University.

Allon, E., Moses, Carlos Hidalgo-Grass, Mary Dan-Goor, et al. 2003. emm Typing of $\mathrm{M}$ Nontypeable Invasive Group A Streptococcal Isolates in Israel, J. Clin. Microbiol., 41(10): 4655-4659.

Ananthanarayan and Paniker's Textbook of Microbiology, ${ }^{7 \text { th }}$ Edition, pg 202-215.

Andrea Brenciani, Alessandro Bacciaglia, Manuela Vecchi, Luca, A., Vitali Pietro E. Varaldo and Eleonora Giovanetti. 2007. Genetic Elements Carrying erm(B) inStreptococcus pyogenes and Association with tet $(\mathrm{M})$ Tetracycline Resistance Gene' Antimicrob. Agents Chemother., vol. 51 no. 4 1209-1216.

Arnold, N., Weinberg, Daniel, J., Sexton. 2012. Group C and Group G Streptococcal Infection, Wolters Kluwer Health.

Ashley Robinson, D., Joyce A. Sutcliffe, Wezenet Tewodros, Anand Manoharan, and Debra, E., Bessen. 2006. Evolution and Global Dissemination of MacrolideResistant Group A Streptococci. Antimicrobial Agents and Chemotherapy, p. 2903-2911.

Ashton, H., Minty. 2012. Information on Beta Strep Group C, eHow health.

Carapetis, J.R., A.C. Steer, E.K. Mulholland and M. Weber. 2005. The global burden of Group A Streptococcal diseases. Lancet Infect, Dis., 5:685-694.

Carmen Franken, Mark Vander Linden, et al. 2004. Real time PCR for detection of Macrolide resistance genes in S. pyogenes and S.pneumoniae in Germany. Int. J. Antimicrobial Agents, Volume 24, Issue 1, Pages 43-47.

Carriço, J.A., C. Silva-Costa, J. Melo-Cristino, F.R. Pinto, H. de Lencastre, J. S. Almeida and M. Ramirez. 2006. Illustration of a Common Framework for Relating Multiple Typing Methods by Application to Macrolide-Resistant Streptococcus pyogenes, J. Clin. Microbiol., vol. 44 no. 7 2524-2532.

Charmaine, A., C. Lloyd, Swarna, E. Jacob and Thangam Menon. 2007. Antibiotic resistant $\beta$-hemolytic Streptococci, Indian J. Pediatrics, Volume 74, Number 12, 1077-1080, Doi: 10.1007/S12098-0070200-1

Chun-Hsing Liao, Liang-Chun Liu, Yu-Tsung Huang, Lee-Jeng Teng and Po-Ren Hsueh. 2008. Bacteremia Caused by GroupG Streptococci, Taiwan, Emerg. Infect. Dis., 14(5): 837-840.

Clancy, J., J. Petitpas, et al. 1996. Molecular cloning and functional analysis of a novel macrolide-resistance determinant, mef A, from Streptococcus pyogenes." Mol. Microbiol., Volume: 22, Issue: 5, Pages: 867-879.

David, J., Farrell*, Ian Morrissey, Sarah Bakker, et al. 2004. Molecular Epidemiology of Multi resistant Streptococcus pneumoniae with Both $\operatorname{erm}(\mathrm{B})$ - and mef(A)-Mediated Macrolide Resistance. J. Clin. Microbiol., vol. 42 no. 2764-768.

Duvuru Geetha. 2010. Acute glomerulonephritis - Post Streptococcal, Medscape.

Edouard Bingen, Frederic Fitoussi et al. 2000. Resistance to Macrolides in Streptococcus pyogenes in France in Pediatric Patients, Antimicrob. Agents Chemother., 44(6): 1453-1457.

Eleonora Giovanetti, Andrea Brenciani, Remo Lupidi, Marilyn C. Roberts and Pietro E. Varaldo,. 2003. Presence of the tet $(\mathrm{O})$ Gene in Erythromycin- and TetracyclineResistant Strains of Streptococcus pyogenes and Linkage with either the $m e f(\mathrm{~A})$ or the erm(A) Gene, Antimicrob. Agents Chemother., vol. 47 no. 928442849.

Emerging infectious disease, Increase in Group $\mathrm{G}$ Streptococcal Infections in a Community Hospital, New York, USA, Center for Disease control, Volume 15, Number 6.

Ergin Ciftci, Ulker Doğru, Haluk Guriz, Ahmet Derya Aysev, Erdal. 2003. Antibiotic 
susceptibility of Streptococcus pyogenes strains isolated from throat cultures of children with tonsillopharyngitis, $J$. Ankara Med. School, Vol 25, No1.

Fernando Baquero, Gregorio Baquero-Artigao, Rafael Cantón and César García-Rey, Antibiotic consumption and resistance selection in Streptococcus pneumoniae, $J$. Antimicrobial Chemother., 50, Suppl. S2, 27-37.

Gabriela Rubinstein, Bárbara Bavdaz, Sabrina De Bunder and Néstor Blazquez. 2011. Trends in Macrolide Resistance for Streptococcus pyogenes, Streptococcus agalactiaeandStreptococcus pneumoniae and its Association with Social Clustering in Argentina, The Open Antimicrobial Agents J., 3: 1-5.

Granizo, J.J., L. Agnilar, J. Casal, et al. 2000. Streptococcus pyogenes to Erythromycin in relation to macrolide consumption in Spain, J. Antimicrobial Chemother., 46: 959-964.

Hofmann, J., Cetron, M.S., Farley, M.M., Baughman, W.S., Facklam, R.R., Elliott, J.A., Deaver, K.A., Breiman, R.F. 1995. "The prevalence of drug-resistant Streptococcus pneumoniae in Atlanta". Pub Med, N. Engl J. Med., 333(8): 481-6.

Ileana Cochetti, Manuela Vecchi, et al. 2005. "Molecular Characterization of Pneumococci with Efflux - Mediated Erythromycin Resistance and Identification of a Novel mef Gene Sub class, mef (I)". Antimicrob. Agents Chemother., 49(12): 4999-5006.

Isabel Garcia-Bermejo and Juana Cacho et al. 1998. Emergence of ErythromycinResistant, Clindamycin-Susceptible Streptococcus pyogenes isolates in Madrid, Spain, Antimicrob. Agents Chemother., vol. 42 no. 4989-990.

Jacob, S.E., C.A.C. Lloyd, T. Menon. 2006. cMLS and M Phenotypes among Streptococcus pyogenes isolates in Chennai, Indian J. Med. Microbiol., Volume: 24 Issue: 2 Page: 147-148.

Jae-Hoon Song, Hyun-Ha Chang, et al. "Macrolide resistance and genotypic characterization of Streptococcus pneumoniae in Asian countries: a study of the Asian Network for Surveillance of Resistant Pathogens (ANSORP)", J. Antimicrobial Chemother., 53: 457-463.

Janne Kataja, Pentti Huovinen, Mikael Skurnik. 1999. "Erythromycin Resistance Genes in Group A Streptococci" in Finland, Antimicrobial Agent and Chemother., 43(1): 48-52.

Jawetz Melnick \& Adelberg's, Textbook of Medical Microbiology, 25 ${ }^{\text {th }}$ Edition, pg 195-206, \& 342.

Jinnethe Reyes, Marylin Hidalgo, Lorena Díaz, Sandra Rincón, Jaime Moreno, Natasha Vanegas, Elizabeth Castañeda, César A. Arias, Characterization Of Macrolide Resistance In Gram-Positive Cocci From Colombian Hospitals: A Countrywide Surveillance, Int. J. Infect. Dis., Volume 11, Issue 4, Pages 329-336.

Juan, J. Granizoa, Lorenzo Aguilarb, Julio Casalc, Rafael Dal-Réb and Fernando Baquerod. 2000. Streptococcus pyogenes resistance to erythromycin in relation to macrolide consumption in Spain (19861997), J. Antimicrob. Chemother., 46(6): 959-964.

Karen Lin, Philip M. Tiernojr, Aarnoldkomisar. 2009. "increasing antibiotic resistance of Streptococcus species in new york city" WILEY online library, Vol 114 Issue 7.

Kenneth Todar, Madison and Wisconsin. 2008. Streptococcus pneumoniae, Online textbook of Bacteriology, Pg 1, 20082012.

Kertesz, D.A., Di Fabio, J.L., de Cunto Brandileone, M.C., Castañeda, E., Echániz-Aviles, G., Heitmann, I., Homma, A., Hortal, M., Lovgren, M., Ruvinsky, R.O., Talbot, J.A., Weekes, J., Spika, J.S. 1998. Invasive Streptococcus pneumoniae infection in Latin American children: results of the Pan American Health Organization Surveillance Study, Pub Med, Clin. Infect. Dis., 26(6): 135561.

Kiran Chawla, Bimala Gurung, Chiranjay Mukhopadhyay, Indira Bairy. 2010. 
Reporting emerging resistance of Streptococcus pneumoniae from India. Kasturba Medical College, Manipal, Karnataka, India, and Year: 2010, Volume: 2, Page: 10-14.

Koneman, Color atlas and Textbook of Diagnostic Microbology, $6^{\text {th }}$ edition.

Louis Davignon, Elizabeth, A., Walter, et al. 2005. Use of $\mathrm{Re}$ sequencing Oligonucleotide Microarrays for Identification of Streptococcus pyogenes and Associated Antibiotic Resistance Determinants, J. Clin. Microbiol., 43(11): 5690-5695

Mark, R., Schleiss. 2010. Group A streptococcal infections ". Medscape.

Matteo Bassetti, GrazianaManno, Andrea Collidà, Alberto Ferrando, Giorgio Gatti, Elisabetta Ugolotti, Mario Cruciani, and Dante Bassetti, Erythromycin Resistance in Streptococcus pyogenes in Italy, Emerging Infect. Dis., CDC, Volume 6, Number 2.

Mazzei, T., E. Mini, A. Novelli and P. Periti. 1993. Chemistry and mode of action of macrolides, J. Antimicrobial Chemother., vol.31, pg 1-9.

National Institute of Allergy and Infectious Disease (NIAID). 2010. U.S Department of Health and Services, National Institutes of Health.

Nguyen, L., D. Levy, A. Ferroni et al. 1997. Molecular epidemiology of Streptococcus pyogenes in an area where actuepharngotonsilliis is Endemic, vol-35, J. Clin. Microbiol., 08: 2111-2114.

Nielsen, H.U., Hammerum, A.M., Ekelund, K., Bang, D., Pallesen, L.V., Frimodt-Møller, N. 2004. Tetracycline and macrolide coresistance in Streptococcus pyogenes: coselection as a reason for increase in macrolide-resistant S. pyogenes, Pub Med, Microb. Drug Resist., 10(3): 231-8.

Pallaval, V., Bramhachari, et al. "Disease burden due to Streptococcus dysgalactiae subsp. equisimilis (group $\mathrm{G}$ and $\mathrm{C}$ Streptococcus) is higher than that due to Streptococcus pyogenes among Mumbai school children", J. Med. Microbiol., 59: 220-223.

Parija, S.C., S. Sujatha, Annie, B., Khyriem. 2002. Simple Diagnostic methods in Infectious Diseases, MIC Method, JIPMER Manual, $3^{\text {rd }}-7^{\text {th }}$ December 2002.

Paul, H. 2011. Edelstein Streptococcal Macrolide Resistance Mechanism, Antimicrobial Agent and Chemother., 1/25/02.Modified4/29/03.

Perez-Trallero, E., et al. 1998. Emergence of Streptococcus pyogenes strains resistant to Erythromycin in Gipuzkoa, Spain, 17: 25-31.

Pérez-Trallero, E., C. Fernández-Mazarrasa, C. García-Rey, E. Bouza, L. Aguilar, J. García-de-Lomas, F. Baquero. 2001. Antimicrobial Susceptibilities of 1,684 Streptococcus pneumoniae and 2,039 Streptococcus pyogenes Isolates and Their Ecological Relationships: Results of a 1-Year (1998-1999) Multicenter Surveillance Study in Spain, Antimicrob. Agents Chemother., 45(12): 3334-3340.

Peter, C., Appelbaum. 1991. "Antimicrobial Resistance in Streptococcus pneumoniae": Clin. Infect. Dis., Volume 15, Issue 1, Pg. 77-83.

Po-renhsueh, Hung-Mochen, Ay-Hueyhuang, and Jiunn-Jong Wu, Decreased Activity of Erythromycin against Streptococcus pyogenes in Taiwan. National Cheng Kung University Medical College,2 Tainan, Taiwan, Republic of China, August 1995.

Ralf René Reinert, Adrian Ringelstein, Mark van der Linden, et al. 2005. "Molecular Epidemiology of Macrolide-Resistant Streptococcus pneumoniae Isolates in Europe", J. Clin. Microbiol., 43(3): 12941300.

Robert, J., Meador. 2011. Acute Rheumatic Fever Treatment and Management, Medscape.

San, S., Wong, Yu, S., Lin, et al. 2009. "Increase in Group G Streptococcal Infections in a Community Hospital", 
New York, USA, CDC, Emerging Infect Dis., Volume 15, Number 6.

Silva-Costa, C. M. Ramirez, J. Melo-Cristino. 2005. Rapid Inversion of the Prevalence's of Macrolide Resistance Phenotypes Paralleled by a Diversification of $\mathrm{T}$ and emm Types among Streptococcus pyogenes in Portugal, Antimicrob. Agents Chemother., vol. 49 no. 5210.

Stephen, G., Jenkins, Steven, D., Brown and David, J. Farrell. 2008. "Trends in antibacterial resistance among Streptococcus pneumoniae isolated in the USA": Annals of Clin. Microbiol. Antimicrobials, 7: 1 .

Thea Horodniceanu, Annie Buu-Hoi, Francoise Delbos, And Gilda Bieth. 1982. HighLevel Aminoglycoside Resistance in Group A, B, G, D (Streptococcus bovis), and Viridans Streptococci, Antimicrobial Agents And Chemother., 176-179.

Theoklis Zaoutis, Barbara Schneider, Lynn Steele Moore, and Joel D. Klein. 1999. "Antibiotic Susceptibilities of Group C and Group G Streptococci Isolated from Patients with Invasive Infections: Evidence of Vancomycin Tolerance among Group G Serotypes", J. Clin. Microbiol., 37(10): 3380-3383.

Van Eldere, J., E. Meekers, et al. 2005. "Macrolide-resistance mechanisms in
Streptococcus pneumoniae isolates" from Belgium, Clin. Microbiol. Infect., Vol 11 Issue 4.

Wayne, Pa. 2010. CLSI clinical and lab standard institute Guide line.

Werner, C., Albrich, Dominique, L., Monnet, and Stephan Harbarth. 2004. Antibiotic Selection Pressure and Resistance in Streptococcus pneumoniae and Streptococcus pyogenes, Emerging Infect. Dis., Volume 10, Number 3.

Wezenet Tewodros and Göran Kronvall, M. 2005. Protein Gene (emm Type) Analysis of Group A Beta-Hemolytic Streptococci from Ethiopia Reveals Unique Patterns, $J$. Clin. Microbiol., vol. 43 no. 9 4369-4376.

Williams, Gail, S. 2003. Group C and G Streptococci infections: Emerging Challenges Clinical laboratory science, $J$. American Society for Med. Technol., Volume: 16, Issue: 4, Pages: 209-213.

Williams, R.E.O. 1958. Laboratory diagnosis of streptococcal infections" PubMed Central, Vol. 19(1): 153-176. Rheumatic fever and rheumatic heart disease. Report of a WHO study group. Geneva, World Health Organization, 1988 (WHO Technical Report Series, No.764).

Zartash Zafar Khan. 2012. Streptococcus Group A infections Medication, Medscape Drug Dis. Procedure.

\section{How to cite this article:}

Illakya, V. Sangamithra, Shabana Praveen and Radha Madhavan. 2017. Prevalence of Betahemolytic Streptococci other than Group A in a Tertiary Health Care Centre. Int.J.Curr.Microbiol.App.Sci. 6(5): 912-924. doi: https://doi.org/10.20546/ijcmas.2017.605.101 